The University of San Francisco

USF Scholarship: a digital repository @ Gleeson Library |

Geschke Center

Mathematics

College of Arts and Sciences

2007

\title{
Vorticity Dynamics and Sound Generation in Two- Dimensional Fluid Flow
}

Raymond J. Nagem

Guido Sandri

David Uminsky

University of San Francisco, duminsky@usfca.edu

Follow this and additional works at: http://repository.usfca.edu/math

Part of the Acoustics, Dynamics, and Controls Commons, and the Mathematics Commons

\section{Recommended Citation}

Raymond J. Nagem, Guido Sandri, and David Uminsky. Vorticity dynamics and sound generation in two-dimensional fluid flow. The Journal of the Acoustical Society of America, 122(1):128-134, 2007.

This Article is brought to you for free and open access by the College of Arts and Sciences at USF Scholarship: a digital repository @ Gleeson Library | Geschke Center. It has been accepted for inclusion in Mathematics by an authorized administrator of USF Scholarship: a digital repository @ Gleeson Library | Geschke Center. For more information, please contact repository@usfca.edu. 


\title{
Vorticity dynamics and sound generation in two-dimensional fluid flow
}

\author{
Raymond J. Nagem ${ }^{\mathrm{a})}$ and Guido Sandri \\ Department of Aerospace and Mechanical Engineering, Boston University, Boston, Massachusetts 02215 \\ David Uminsky \\ Department of Mathematics and Statistics, Boston University, Boston, Massachusetts 02215
}

(Received 12 December 2006; revised 10 April 2007; accepted 12 April 2007)

\begin{abstract}
An approximate solution to the two-dimensional incompressible fluid equations is constructed by expanding the vorticity field in a series of derivatives of a Gaussian vortex. The expansion is used to analyze the motion of a corotating Gaussian vortex pair, and the spatial rotation frequency of the vortex pair is derived directly from the fluid vorticity equation. The resulting rotation frequency includes the effects of finite vortex core size and viscosity and reduces, in the appropriate limit, to the rotation frequency of the Kirchhoff point vortex theory. The expansion is then used in the low Mach number Lighthill equation to derive the far-field acoustic pressure generated by the Gaussian vortex pair. This pressure amplitude is compared with that of a previous fully numerical simulation in which the Reynolds number is large and the vortex core size is significant compared to the vortex separation. The present analytic result for the far-field acoustic pressure is shown to be substantially more accurate than previous theoretical predictions. The given example suggests that the vorticity expansion is a useful tool for the prediction of sound generated by a general distributed vorticity field. (C) 2007 Acoustical Society of America. [DOI: 10.1121/1.2736513]
\end{abstract}

PACS number(s): 43.28.Ra, 43.20.Rz [RMW]

Pages: $128-134$

\section{INTRODUCTION}

The prediction of aerodynamic sound depends on establishing a connection between an unsteady fluid flow, which is most commonly modeled as incompressible, and the small compressible fluctuations associated with an acoustic field. The most successful approach to this fundamentally unsolved problem has identified the vorticity of the incompressible fluid flow as an important source for the generation of sound. This theory, which takes its starting point from the Lighthill acoustic analogy, ${ }^{1}$ has provided many examples of sound generation by a given vorticity distribution in a fluid flow. $^{2}$

In this paper, we begin in Sec. II with the general equation for the time evolution of the vorticity in a twodimensional incompressible flow. We expand the vorticity field in a series of derivatives of a Gaussian vortex. We then derive a set of ordinary differential equations for the timedependent coefficients of our series. A solution of these ordinary differential equations provides a solution for the timedependent vorticity and velocity fields. In Sec. III, we apply our method to analyze the motion of two corotating Gaussian vortices, and show that the second-order term of our vorticity expansion rotates in space with a frequency which reduces, as the vortex core size and the viscosity approach zero, to the rotation frequency predicted by the Kirchhoff point vortex theory. In Sec. IV, we show how our solution of the vorticity equation can be used in the Lighthill equation for sound generation. We use the Lighthill equation to compute the sound produced by the Gaussian vortex pair, and compare

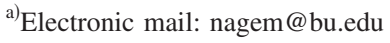

our result to a previous high Reynolds number numerical computation which is based on a numerical solution of the compressible Navier-Stokes equations. It is shown that our analytic result for the far-field pressure is substantially more accurate than previous theoretical predictions.

Our example suggests that the second-order term of our vorticity expansion is sufficient to provide a good approximation to the sound generated by a two-dimensional distributed vorticity field. The higher-order terms in our expansion, which are not considered in detail here, may give additional insight into the general structure (for example, vortex core distortion and vortex core interaction) of two-dimensional vortex dynamics.

\section{VORTICITY DYNAMICS FOR TWO-DIMENSIONAL INCOMPRESSIBLE FLOW}

The continuity and momentum equations for twodimensional incompressible fluid flow can be written in the form

$$
\begin{aligned}
& \frac{\partial u_{i}}{\partial x_{i}}=0, \\
& \dot{\omega}+u_{i} \frac{\partial \omega}{\partial x_{i}}=\nu \frac{\partial^{2} \omega}{\partial x_{i} \partial x_{i}},
\end{aligned}
$$

where $u_{i}\left(x_{1}, x_{2}, t\right)$ is the fluid velocity vector, $\nu$ is the kinematic viscosity, and 


$$
\omega\left(x_{1}, x_{2}, t\right)=\varepsilon_{j k} \frac{\partial u_{k}}{\partial x_{j}}, \quad \varepsilon_{12}=-\varepsilon_{21}=1, \quad \varepsilon_{11}=\varepsilon_{22}=0
$$

is the single component of the fluid vorticity. Throughout this paper, the superposed dot indicates the time derivative, alphabetic subscripts take the value 1 or 2 , and the summation convention for repeated subscripts is assumed.

A particular solution of Eqs. (1)-(3) is the Gaussian vortex ${ }^{3}$ defined by the vorticity and velocity fields

$$
\omega=\gamma \delta^{\lambda}\left(x_{1}, x_{2}, t\right) \equiv \gamma \frac{e^{-r^{2} / \lambda^{2}}}{\pi \lambda^{2}}
$$

and

$$
u_{i}=\gamma U_{i}^{\lambda}\left(x_{1}, x_{2}, t\right) \equiv \gamma \frac{1-e^{-r^{2} / \lambda^{2}}}{2 \pi r} \hat{\theta}_{i}=\gamma \frac{1-e^{-r^{2} / \lambda^{2}}}{2 \pi r}\left(-\varepsilon_{i j} \hat{r}_{j}\right)
$$

where

$$
\lambda=\sqrt{\lambda_{0}^{2}+4 \nu t}=\lambda_{0} \sqrt{1+4 \nu t / \lambda_{0}^{2}},
$$

$r=\sqrt{x_{1}^{2}+x_{2}^{2}}$, and $\hat{r}_{i}, \hat{\theta}_{i}$ are the $i$ th components of the radial and tangential unit vectors corresponding to the $r, \theta$ polar coordinate system in the $x_{1}, x_{2}$ plane. The vorticity distribution $\gamma \delta^{\lambda}(r, t)$ is a two-dimensional radially symmetric Gaussian whose total integral over the $x_{1}, x_{2}$ plane is equal to the constant $\gamma$. The spatial broadness of the Gaussian at time $t$ $=0$ is characterized by the constant $\lambda_{0}$, and the spatial broadness $\lambda(t)$ increases in proportion to $\sqrt{t}$. As $\lambda \rightarrow 0$, $\gamma \delta^{\lambda}(r, t)$ approaches the two-dimensional point vortex $\gamma \delta(\mathbf{r})$. The tangential velocity $\gamma U^{\lambda}$ is zero at $r=0$, and has a radially symmetric core region in which the tangential velocity increases to a maximum value of approximately $(0.102) \gamma / \lambda$ at a radius of approximately (1.121) $\lambda$. For $r \gg \lambda$, the tangential velocity $\gamma U^{\lambda}$ approaches the value $\gamma / 2 \pi r$ associated with the point vortex $\gamma \delta(\mathbf{r})$.

To obtain a more general solution of Eqs. (1)-(3), we construct the series

$$
\begin{aligned}
& \omega=\Gamma(t) \delta^{\lambda}+D_{j}(t) \frac{\partial}{\partial x_{j}} \delta^{\lambda}+Q_{j k}(t) \frac{\partial^{2}}{\partial x_{j} \partial x_{k}} \delta^{\lambda}+\cdots, \\
& u_{i}=\Gamma(t) U_{i}^{\lambda}+D_{j}(t) \frac{\partial}{\partial x_{j}} U_{i}^{\lambda}+Q_{j k}(t) \frac{\partial^{2}}{\partial x_{j} \partial x_{k}} U_{i}^{k}+\cdots,
\end{aligned}
$$

which satisfy Eqs. (1) and (3) identically. The series coefficients $\Gamma(t), D_{j}(t)$, and $Q_{j k}(t)$ are to be determined by vorticity equation (2). The coefficient $Q_{j k}$ is assumed, without loss of generality, to be symmetric in $j$ and $k$. A similar series for the vorticity and velocities fields has been used previously to derive long-time asymptotics of the two-dimensional NavierStokes and vorticity equations. ${ }^{4}$

The series (7) is a convergent expansion in the twodimensional Gaussian derivatives, a complete and orthogonal set of spatial basis functions for functions which have spatial moments of all orders. ${ }^{5}$ Using the orthogonality property of these basis functions, the first three time-dependent series coefficients in Eq. (7) are given in terms of $\omega$ by the moments

$$
\begin{aligned}
& \Gamma=\iint d^{2} \mathbf{r} \omega, \\
& D_{j}=-\iint d^{2} \mathbf{r} x_{j} \omega, \\
& Q_{j k}=\iint d^{2} \mathbf{r}\left(\frac{x_{j} x_{k}}{2}-\frac{\lambda^{2} \delta_{j k}}{4}\right) \omega,
\end{aligned}
$$

where $\delta_{j k}$ is the Kronecker delta.

We refer to the successive terms on the right-hand side of Eq. (7) as the monopole, dipole, and quadrupole components of the vorticity field. This terminology is conventional when the two-dimensional Dirac delta function $\delta(\mathbf{r})$ is used in place of the Gaussian $\delta^{\lambda}(r, t)$. The corresponding terminology is used for the successive terms in the velocity field given by Eq. (8). By using $\delta^{\lambda}(r, t)$ instead of $\delta(\mathbf{r})$, we avoid the singularities associated with the distribution $\delta(\mathbf{r})$ and, as shown in Eq. (14), simultaneously account for the viscous diffusion that is explicitly built into the function $\delta^{\lambda}(r, t)$ via the parameter $\lambda$ defined in Eq. (6).

Substitution of the series (7) and (8) into Eq. (2) gives

$$
\begin{aligned}
\dot{\Gamma} \delta^{\lambda}+ & \Gamma \dot{\delta}^{\lambda}+\dot{D}_{j} \frac{\partial \delta^{\lambda}}{\delta x_{j}}+D_{j} \frac{\partial \dot{\delta}^{\lambda}}{\partial x_{j}}+\dot{Q}_{j k} \frac{\partial^{2} \delta^{\lambda}}{\partial x_{j} \partial x_{k}}+Q_{j k} \frac{\partial^{2} \dot{\delta}^{\lambda}}{\partial x_{j} \partial x_{k}} \\
& +\cdots+\frac{\partial}{\partial x_{i}}\left[\left(\Gamma U_{i}^{\lambda}+D_{j} \frac{\partial U_{i}^{\lambda}}{\partial x_{j}}+Q_{j k} \frac{\partial^{2} V_{i}^{\lambda}}{\partial x_{j} \partial x_{k}}+\cdots\right)\right. \\
& \left.\times\left(\Gamma \delta^{\lambda}+D_{m} \frac{\partial \delta^{\lambda}}{\partial x_{m}}+Q_{m n} \frac{\partial^{2} \delta^{\lambda}}{\partial x_{m} \partial x_{n}}+\cdots\right)\right] \\
= & \nu \frac{\partial^{2}}{\partial x_{i} \partial x_{i}}\left(\Gamma \delta^{\lambda}+D_{j} \frac{\partial \delta^{\lambda}}{\partial x_{j}}+Q_{j k} \frac{\partial^{2} \delta^{\lambda}}{\partial x_{j} \partial x_{k}}+\cdots\right)
\end{aligned}
$$

where we have used

$$
u_{i} \frac{\partial \omega}{\partial x_{i}}=\frac{\partial}{\partial x_{i}}\left(u_{i} \omega\right)
$$

on the basis of Eq. (1). Since

$$
\dot{\delta}^{\lambda}=\nu \frac{\partial^{2}}{\partial x_{i} \partial x_{i}} \delta^{\lambda}
$$

Eq. (12) reduces to the much simpler equation

$$
\begin{aligned}
\dot{\Gamma} \delta^{\lambda} & +\dot{D}_{j} \frac{\partial \delta^{\lambda}}{\partial x_{j}}+\dot{Q}_{j k} \frac{\partial^{2} \delta^{\lambda}}{\partial x_{j} \partial x_{k}}+\cdots+\frac{\partial}{\partial x_{i}}\left[\left(\Gamma U_{i}^{\lambda}+D_{j} \frac{\partial U_{i}^{\lambda}}{\partial x_{j}}\right.\right. \\
& \left.+Q_{j k} \frac{\partial^{2} U_{i}^{\lambda}}{\partial x_{j} \partial x_{k}}+\cdots\right)\left(\Gamma \delta^{\lambda}+D_{m} \frac{\partial \delta^{\lambda}}{\partial x_{m}}+Q_{m n} \frac{\partial^{2} \delta^{\lambda}}{\partial x_{m} \partial x_{n}}\right. \\
& +\cdots)]=0 .
\end{aligned}
$$

Differential equations for the temporal functions $\Gamma(t)$, $D_{j}(t)$, and $Q_{j k}(t)$ can now be obtained from Eq. (15) by using the orthogonality properties of the two-dimensional Gaussian derivatives, as discussed earlier in conjunction with Eqs. (9)-(11). Integration of Eq. (15) over the entire $x_{1}, x_{2}$ plane gives 


$$
\dot{\Gamma}=-\iint d^{2} \mathbf{r} \frac{\partial}{\partial x_{i}}\left(u_{i} \omega\right),
$$

which after an integration by parts gives

$$
\dot{\Gamma}=0
$$

The coefficient $\Gamma$, according to Eq. (9), is the total spatial integral of the vorticity field. Equation (17) thus states the known result that this total spatial integral of the vorticity is constant in time.

Taking the first spatial moment of Eq. (15) gives

$$
\dot{D}_{j}=\iint d^{2} \mathbf{r} x_{j} \frac{\partial}{\partial x_{i}}\left(u_{i} \omega\right)=-\delta_{i j} \iint d^{2} \mathbf{r} u_{i} \omega
$$

where the last equality is obtained by an integration by parts. It is shown in Appendix A that the integral on the right-hand side of Eq. (18) vanishes, so that

$$
\dot{D}_{j}=0 \text {. }
$$

We thus obtain the known result that the dipole coefficient $D_{j}$ in the series (7), which according to Eq. (10) is the negative of the first spatial moment of the vorticity field, is also constant in time.

The time derivative of the quadrupole coefficient $Q_{j k}$ in Eq. (7) is obtained from Eq. (15) as

$$
\begin{aligned}
\dot{Q}_{p q}= & -\iint d^{2} \mathbf{r}\left(\frac{x_{j} x_{k}}{2}-\frac{\lambda^{2} \delta_{j k}}{4}\right)\left\{\frac { \partial } { \partial x _ { i } } \left[\left(\Gamma U_{i}^{\lambda}+D_{p} \frac{\partial U_{i}^{\lambda}}{\partial x_{p}}\right.\right.\right. \\
& \left.+Q_{p q} \frac{\partial^{2} U_{i}^{\lambda}}{\partial x_{p} \partial x_{q}}+\cdots\right) \times\left(\Gamma \delta^{\lambda}+D_{m} \frac{\partial \delta^{\lambda}}{\partial x_{m}}\right. \\
& \left.\left.\left.+Q_{m n} \frac{\partial^{2} \delta^{\lambda}}{\partial x_{m} \partial x_{n}}+\cdots\right)\right]\right\} .
\end{aligned}
$$

Each term in the infinite series on the right-hand side of Eq. (20) can be integrated in closed form. We have evaluated the terms up the quadrupole order explicitly; the result of these evaluations is

$$
\begin{aligned}
\dot{Q}_{j k}= & -\frac{Q_{i i}}{8 \pi \lambda^{4}}\left(Q_{j m} \varepsilon_{m k}+Q_{k m} \varepsilon_{m j}\right)+\frac{\Gamma}{8 \pi \lambda^{2}}\left(Q_{j m} \varepsilon_{m k}\right. \\
& \left.+Q_{k m} \varepsilon_{m j}\right)+\frac{1}{16 \pi \lambda^{2}}\left(D_{j} D_{m} \varepsilon_{k m}+D_{k} D_{m} \varepsilon_{j m}\right) .
\end{aligned}
$$

If we set $j=k$ in Eq. (21), we obtain

$$
\dot{Q}_{k k}=0 \text {, }
$$

so that

$$
T=Q_{k k}=Q_{11}+Q_{22}=\operatorname{Tr}(Q)=\text { constant } .
$$

Equations (21)-(23) are based on a truncation of the series (7) and (8), but it can be shown directly from Eq. (11) (see Appendix B) that the result $\operatorname{Tr}(Q)=$ constant remains valid when the entire series (7) and (8) are retained.

The constants $\Gamma$ and $D_{j}$, together with a solution of Eq. (21), give, via Eqs. (7) and (8), an approximate solution for the vorticity and velocity fields. The values of the constants $\Gamma$ and $D_{j}$ and the initial values of $Q_{j k}$ are obtained in terms of the initial value of the vorticity distribution by means of Eqs. (9)-(11). At $t=0$, the parameter $\lambda$ reduces to the constant $\lambda_{0}$, which is chosen on the basis of the length scale associated with the initial vorticity distribution.

\section{MOTION OF A GAUSSIAN VORTEX PAIR} tion

As an example, we consider the initial vorticity distribu-

$$
\omega(x, y, 0)=\gamma \delta^{a}\left(x_{1}-\ell, x_{2}\right)+\gamma \delta^{a}\left(x_{1}+\ell, x_{2}\right),
$$

where the function $\delta^{a}$ is defined by Eq. (4) with $\lambda$ equal to the constant value $a$. Equation (24) represents two equal corotating Gaussian vortices on the $x_{1}$ axis, separated by the distance $2 \ell$. The initial core size of the vortices is determined by the parameter $a$. If the core size $a$ is zero, the Gaussians become point vortices. In the absence of viscosity, the Kirchhoff theory of two-dimensional point vortex motion ${ }^{6}$ predicts that two such point vortices will rotate around the origin of the $x_{1}, x_{2}$ plane on a circle of radius $2 \ell$ with angular velocity $\gamma / 4 \pi \ell^{2}$. We will use the series (7) to predict the approximate time evolution of the Gaussian vortices, including the effects of viscosity and the finite size of the vortex core regions.

For the initial vorticity distribution (24), Eqs. (9)-(11) give, with $\lambda_{0}=\ell$, the monopole, dipole, and and initial quadrupole coefficients,

$$
\begin{aligned}
& \Gamma=2 \gamma, \\
& D_{1}=D_{2}=0, \\
& Q_{11}(0)=\gamma\left(\frac{a^{2}}{2}+\frac{\ell^{2}}{2}\right), \\
& Q_{12}(0)=0, \\
& Q_{22}(0)=\gamma\left(\frac{a^{2}}{2}-\frac{\ell^{2}}{2}\right) .
\end{aligned}
$$

Here

$$
T=Q_{11}+Q_{22}=\gamma a^{2},
$$

and the components of Eq. (21) become

$$
\begin{aligned}
& \dot{Q}_{11}=\frac{Q_{12}}{4 \pi \lambda^{2}}\left(\frac{T}{\lambda^{2}}-\Gamma\right), \\
& \dot{Q}_{12}=-\frac{Q_{11}-Q_{22}}{8 \pi \lambda^{2}}\left(\frac{T}{\lambda^{2}}-\Gamma\right), \\
& \dot{Q}_{22}=-\frac{Q_{12}}{4 \pi \lambda^{2}}\left(\frac{T}{\lambda^{2}}-\Gamma\right) .
\end{aligned}
$$

Letting

$$
S=Q_{11}-Q_{22},
$$

Eqs. (31)-(33) imply that

$$
\dot{S}=\frac{Q_{12}}{2 \pi \lambda^{2}}\left(\frac{T}{\lambda^{2}}-\Gamma\right),
$$




$$
\dot{Q}_{12}=-\frac{S}{8 \pi \lambda^{2}}\left(\frac{T}{\lambda^{2}}-\Gamma\right)
$$

which in turn imply that

$$
-4 Q_{12} \dot{Q}_{12}=S \dot{S},
$$

or

$$
S^{2}+4 Q_{12}^{2}=4 C_{1}^{2},
$$

where $C_{1}$ is a constant. Equations (36) and (38) now give

$$
\frac{d Q_{12}}{\sqrt{4 C_{1}^{2}-4 Q_{12}^{2}}}=\frac{d t}{8 \pi \lambda^{2}}\left(\frac{T}{\lambda^{2}}-\Gamma\right) .
$$

Equation (39) can be integrated to give $Q_{12}$, and Eq. (36) then gives $S$. Using Eq. (23), the results for the quadrupole coefficients are, assuming $C_{1} \neq 0$,

$$
\begin{aligned}
Q_{11}= & \frac{Q_{11}(0)+Q_{22}(0)}{2}+\frac{Q_{11}(0)-Q_{22}(0)}{2} \cos 2 \Omega t \\
& +Q_{12}(0) \sin 2 \Omega t, \\
Q_{12}= & Q_{12}(0) \cos 2 \Omega t+\frac{Q_{11}(0)-Q_{22}(0)}{2} \sin 2 \Omega t, \\
Q_{22}= & \frac{Q_{11}(0)+Q_{22}(0)}{2}-\frac{Q_{11}(0)-Q_{22}(0)}{2} \cos 2 \Omega t \\
& -Q_{12}(0) \sin 2 \Omega t,
\end{aligned}
$$

where the time-dependent frequency $\Omega$ is given by

$$
\Omega=\frac{1}{8 \pi}\left[\frac{\Gamma}{4 \nu t} \ln \left(1+\frac{4 \nu t}{\lambda_{0}^{2}}\right)-\frac{T}{\lambda_{0}^{4}} \frac{1}{1+4 \nu t / \lambda_{0}^{2}}\right] .
$$

For the initial conditions of our example, Eqs. (40)-(44) reduce to

$$
\begin{aligned}
& Q_{11}=\frac{\gamma a^{2}}{2}+\frac{\gamma \ell^{2}}{2} \cos 2 \Omega t, \\
& Q_{12}=\frac{\gamma \ell^{2}}{2} \sin 2 \Omega t, \\
& Q_{22}=\frac{\gamma a^{2}}{2}-\frac{\gamma \ell^{2}}{2} \cos 2 \Omega t, \\
& \Omega=\frac{1}{8 \pi}\left[\frac{\gamma}{2 \nu t} \ln \left(1+\frac{4 \nu t}{\ell^{2}}\right)-\frac{\gamma a^{2}}{\ell^{4}} \frac{1}{1+4 \nu t / \ell^{2}}\right] .
\end{aligned}
$$

Equations (40)-(42) can be written as

$$
\begin{aligned}
{\left[\begin{array}{ll}
Q_{11} & Q_{12} \\
Q_{12} & Q_{22}
\end{array}\right]=} & {\left[\begin{array}{cc}
\cos \Omega t & -\sin \Omega t \\
\sin \Omega t & \cos \Omega t
\end{array}\right]\left[\begin{array}{ll}
Q_{11}(0) & Q_{12}(0) \\
Q_{12}(0) & Q_{22}(0)
\end{array}\right] } \\
& \times\left[\begin{array}{cc}
\cos \Omega t & \sin \Omega t \\
-\sin \Omega t & \cos \Omega t
\end{array}\right]
\end{aligned}
$$

Equation (48) has the familiar form of the transformation equation for a second-rank tensor under a rotation of spatial coordinates. The quadrupole term in the vorticity expansion (7) rotates in space at the frequency $\Omega$. For small viscosity $\nu$, the frequency $\Omega$ in Eq. (43) decreases slowly with time. In the inviscid point vortex limit $\nu \rightarrow 0$ and $a \rightarrow 0$, the rotation frequency $\Omega$ reduces to the Kirchhoff theory value

$$
\Omega=\frac{\gamma}{4 \pi \ell^{2}} .
$$

Thus, the quadrupole term in our vorticity expansion (7) is sufficient to capture the rotation associated with the Kirchhoff point vortex model. For finite vortex core size $a$ and nonzero viscosity $\nu$, the quadrupole term of our expansion also captures the modification of the rotation frequency due to the finite core size, the viscous retardation of the rotation frequency, and the viscous diffusion of the vorticity field.

We note that Eq. (48) is the solution of Eq. (21) for any initial conditions that imply $D_{j}=0$. For $D_{j} \neq 0$, the solution of Eq. (21) consists of Eq. (48) plus a particular solution corresponding to the nonhomogeneous dipole term.

\section{LIGHTHILL EQUATION FOR SOUND GENERATION}

The Lighthill equation for sound generated by a homentropic low Mach number fluid flow is ${ }^{2}$

$$
\left(\frac{1}{c_{0}^{2}} \frac{\partial^{2}}{\partial t^{2}}-\nabla^{2}\right) p=\rho_{0} \frac{\partial^{2}\left(u_{i} u_{j}\right)}{\partial x_{i} \partial x_{j}}
$$

where $p$ is the acoustic pressure perturbation, $\rho_{0}$ is the density of the undisturbed fluid, $c_{0}$ is the speed of sound in the undisturbed fluid, and $u_{i}$ is the incompressible component of the fluid velocity vector. The source term on the right-hand side of Eq. (50) can be written as

$$
S=\rho_{0} \frac{\partial^{2}\left(u_{i} u_{j}\right)}{\partial x_{i} \partial x_{j}}=\rho_{0} \boldsymbol{\nabla} \cdot(\boldsymbol{\omega} \times \mathbf{u})+\rho_{0} \boldsymbol{\nabla}^{2}\left(\frac{1}{2} u^{2}\right),
$$

where $\boldsymbol{\omega}$ is the vorticity vector corresponding to the velocity vector $\mathbf{u}$. It is argued in Ref. 2 that the first term on the right-hand side of Eq. (51) is the principal source of sound at low Mach numbers. Our series expansions for $\boldsymbol{\omega}$ and $\mathbf{u}$ in Sec. II can therefore be used directly in the Lighthill equation to compute the sound generated by a two-dimensional vorticity field. We will use the Lighthill equation to compute the sound produced by the Gaussian vortex pair in the previous section, and compare our result to a previous result ${ }^{2,7}$ which assumes the Kirchhoff theory of point vortex motion.

In order to obtain a far-field solution for the acoustic pressure $p$ in Eq. (50), we expand the principal source term on the right-hand side as

$$
\begin{aligned}
S= & \rho_{0} \boldsymbol{\nabla} \cdot(\boldsymbol{\omega} \times \mathbf{u})=S^{(0)}(t) \delta(\mathbf{r})+S_{p}^{(1)}(t) \frac{\partial}{\partial x_{p}} \delta(\mathbf{r})+S_{p q}^{(2)}(t) \\
& \times \frac{\partial^{2}}{\partial x_{p} \partial x_{q}} \delta(\mathbf{r})+\cdots,
\end{aligned}
$$

where

$$
\begin{aligned}
& S^{(0)}(t)=\iint d^{2} \mathbf{r} S, \\
& S_{p}^{(1)}(t)=-\iint d^{2} \mathbf{r} x_{p} S,
\end{aligned}
$$




$$
S_{p q}^{(2)}(t)=\iint d^{2} \mathbf{r} \frac{x_{p} x_{q}}{2} S .
$$

For the Gaussian vortex pair, the coefficients $S^{(0)}, S_{p}^{(1)}$, and $S_{p q}^{(2)}$ in Eqs. (53)-(55) become

$$
\begin{aligned}
& S^{(0)}=0, \\
& S_{p}^{(1)}=0, \\
& S_{p q}^{(2)}(t)= \\
& \quad \frac{\rho_{0} \delta_{p q}}{24 \pi}\left(-3 \Gamma^{2}+\frac{3 \Gamma Q_{k k}}{\lambda^{2}}-\frac{\left(Q_{k k}\right)^{2}}{\lambda^{4}}-\frac{Q_{k l} Q_{k l}}{12 \pi \lambda^{4}}\right) \\
& \quad+\rho_{0}\left(-\frac{\Gamma Q_{p q}}{4 \pi \lambda^{2}}+\frac{Q_{k k} Q_{p q}}{12 \pi \lambda^{4}}+\frac{Q_{p k} Q_{k q}}{6 \pi \lambda^{4}}\right),
\end{aligned}
$$

where $\Gamma$ and $Q_{j k}$ are given for this example by Eqs. (25) and (44)-(46), respectively. The terms proportional to $\delta_{p q}$ in Eq. (58) are nonoscillatory in time, and thus will not contribute to a propagating sound field. (50) is $^{2}$

The solution of the three-dimensional wave equation

$$
\begin{aligned}
p(\mathbf{R}, t) & =\iiint d^{3} \mathbf{R}^{\prime} \frac{S\left(\mathbf{R}^{\prime}, t-\left|\mathbf{R}-\mathbf{R}^{\prime}\right| / c_{0}\right)}{4 \pi\left|\mathbf{R}-\mathbf{R}^{\prime}\right|} \\
& =\frac{\partial^{2}}{\partial x_{p} \partial x_{q}} \int_{-\infty}^{\infty} d x_{3}^{\prime} \frac{S_{p q}^{(2)}\left(t-\sqrt{x_{1}^{2}+x_{2}^{2}+\left(x_{3}-x_{3}^{\prime}\right)^{2}} / c_{0}\right)}{4 \pi \sqrt{x_{1}^{2}+x_{2}^{2}+\left(x_{3}-x_{3}^{\prime}\right)^{2}}},
\end{aligned}
$$

where $\mathbf{R}=\left(x_{1}, x_{2}, x_{3}\right), \mathbf{R}^{\prime}=\left(x_{1}^{\prime}, x_{2}^{\prime}, x_{3}^{\prime}\right)$, and the source $S$ defined by Eqs. (51) and (52) has been used. For small viscosity, the source expansion term $S_{p q}^{(2)}(t)$ defined by Eqs. (58) and (44)-(46) contains sinusoidal functions which vary slowly in frequency and amplitude. The method of stationary phase can thus be used to evaluate the far-field value of the integral in Eq. (59). Evaluation of Eq. (59) for each of the three oscillatory terms in the second line of Eq. (58) gives

$$
\begin{aligned}
p= & \frac{\rho_{0} \gamma^{2} \ell^{2}}{4 \pi^{2}} \frac{\Omega^{* 2}}{c_{0}^{2} \lambda^{* 2}}\left(\frac{\pi c_{0}}{\Omega^{*} r}\right)^{1 / 2} \cos \left[2 \Omega^{*}\left(t-\frac{r}{c_{0}}\right)-2 \theta-\frac{\pi}{4}\right] \\
& -\frac{a^{2}}{\lambda^{* 2}} \frac{\rho_{0} \gamma^{2} \ell^{2}}{8 \pi^{2}} \frac{\Omega^{* 2}}{c_{0}^{2} \lambda^{* 2}}\left(\frac{\pi c_{0}}{\Omega^{*} r}\right)^{1 / 2} \cos \left[2 \Omega^{*}\left(t-\frac{r}{c_{0}}\right)-2 \theta\right. \\
& \left.-\frac{\pi}{4}\right] .
\end{aligned}
$$

In Eq. (60), $\Omega^{*}$ denotes $\Omega$ evaluated at the retarded time $t$ $-r / c_{0}$, and $\lambda^{*}$ denotes $\lambda$ evaluated at $t-r / c_{0}$. In the inviscid point vortex limit $\nu \rightarrow 0$ and $a \rightarrow 0$, the first term on the righthand side of Eq. (60) reduces to the previous result in Refs. 2 and 7. The second term on the right-hand side of Eq. (60), which comes from the second and third terms in the second line of Eq. (58), is a correction due to the finite vortex core size $a$.

To test the accuracy of our result for the far-field acoustic pressure, we compare our formula with a computational result presented in Ref. 8. In Ref. 8, the far-field acoustic pressure generated by a corotating Gaussian vortex pair is computed by a direct numerical simulation of the compress-
TABLE I. Comparison of results for the amplitude of the nondimensional acoustic pressure produced by a corotating Gaussian vortex pair $\left(k_{0} r=22.6\right.$, $\left.80<t c_{0} / \ell<120\right)$.

\begin{tabular}{ccc}
\hline \hline & $\begin{array}{c}\text { Nondimensional } \\
\text { pressure amplitude }\end{array}$ & \% error \\
\hline Full numerical simulation (Ref. 8) & 0.0014 & $\ldots$ \\
Kirchhoff-Powell (Ref. 7) & 0.0023 & 64 \\
Present work & 0.0017 & 21 \\
\hline \hline
\end{tabular}

ible Navier-Stokes equations. In our notation, case two of Ref. 8 corresponds to the following parameter values:

$$
\operatorname{Re}=\frac{\gamma}{\nu}=226 \times 10^{3}, \quad M_{0}=\frac{\frac{\gamma}{2 \pi a}}{c_{0}}=0.56, \quad \frac{a}{\ell}=0.402 .
$$

Numerical results are given in Ref. 8 for the nondimensional pressure $p / \rho_{0} c_{0}^{2}$ as a function of the nondimensional time $t c_{0} / \ell$, at a distance $r=63 \ell$ from the origin. This value of $r$ corresponds to 3.6 acoustic wavelengths, or $k_{0} r=22.6$, which is a reasonable far-field distance for a numerical computation. Results for $p / \rho_{0} c_{0}^{2}$ are given in Ref. 8 for the time interval $40<t c_{0} / \ell<120$. For $80<t c_{0} / \ell<120$, the transients associated with the initialization of the computation have vanished, and the computed nondimensional pressure is very nearly sinusoidal. The amplitude of this sinusoidal pressure can be compared with that predicted by our Eq. (60).

For the numerical values given in Eq. (61), the dimensional viscous parameter $4 \nu t / \lambda_{0}^{2}=4 \nu t / \ell^{2}$ which appears in Eqs. (6) and (47), satisfies the inequality

$$
\frac{4 \nu t}{\ell^{2}}=\frac{8 \pi}{\operatorname{Re}} \frac{t c_{0}}{\ell} \frac{a}{\ell} M_{0}<3 \times 10^{-3} .
$$

The term $4 \nu t / \ell_{0}^{2}$ is thus negligible in Eqs. (6) and (47), and viscous effects are unimportant in Eq. (60). Therefore,

$$
\lambda^{*} \approx \lambda_{0}=\ell
$$

and

$$
\Omega^{*} \approx \frac{\gamma}{4 \pi \ell^{2}}\left(1-\frac{a^{2}}{2 \ell^{2}}\right) .
$$

The numerical value of the nondimensional pressure amplitude given by Eq. (60) is compared to the computational result of Ref. 8 in Table I. Table I also shows the KirchhoffPowell result, obtained by using the Kirchhoff point vortex theory in the source $S$ defined by Eq. (52), which is equivalent to Eq. (60) with $\nu=a=0$. Möhring's equation for aeroacoustic sound, discussed in detail in Ref. 8, gives a result which is essentially the same as Kirchhoff-Powell value. It is seen from Table I that our result is substantially more accurate than previous theoretical aeroacoustic predictions.

\section{CONCLUSION}

Our result for the corotating Gaussian vortex pair shows that our vorticity expansion can be used in conjunction with the Lighthill equation to provide an analytic prediction of 
vortex generated sound that is substantially more accurate than previous theoretical results. Viscosity is unimportant for the case that we have considered, so our improvement over previous theory must be attributed to the fact that our expansion (7) makes direct use of the vorticity equation (2) to derive the dynamics of the Gaussian vortices with finite core size. While we obviously do not capture all the fluid phenomena included in the numerical computation of Ref. 8, the result of our example suggests that our expansion is a generally useful tool for the prediction of sound generated by a distributed vorticity field.

\section{ACKNOWLEDGMENTS}

The authors wish to thank Professor C. E. Wayne for many valuable and enjoyable discussions. The research of David Uminsky is partially supported by NSF grant DMS0405724.

\section{APPENDIX A: PROOF THAT THE DIPOLE COEFFICIENT $D_{j}$ IS CONSTANT IN TIME}

According to Eq. (18), the time derivative of the dipole coefficient $D_{j}(t)$ is

$$
\dot{D}_{j}=-\delta_{i j} \iint d^{2} \mathbf{r} u_{i} \omega
$$

To evaluate this integral, we introduce the stream function $\psi$, which is related to the velocity vector $u_{i}$ and the vorticity $\omega$ by

$$
u_{i}=\varepsilon_{i k} \frac{\partial \psi}{\partial x_{k}}, \quad \omega=-\frac{\partial^{2} \psi}{\partial x_{m} \partial x_{m}} .
$$

Thus,

$$
\begin{aligned}
\dot{D}_{j} & =\varepsilon_{j k} \iint d^{2} \mathbf{r} \frac{\partial \psi}{\partial x_{k}} \frac{\partial^{2} \psi}{\partial x_{m} \partial x_{m}}=-\varepsilon_{j k} \iint d^{2} \mathbf{r} \frac{\partial^{2} \psi}{\partial x_{k} \partial x_{m}} \frac{\partial \psi}{\partial x_{m}} \\
& =-\varepsilon_{j k} \iint d^{2} \mathbf{r} \frac{\partial}{\partial x_{k}}\left(\frac{1}{2} \frac{\partial \psi}{\partial x_{m}} \frac{\partial \psi}{\partial x_{m}}\right)=0 .
\end{aligned}
$$

The integration by parts and the final equality in Eq. (A3) are valid because the velocity $u_{i}=\partial \psi / \partial x_{i}$ decays at least as fast as $1 / r$ as $r \rightarrow \infty$.

\section{APPENDIX B: PROOF THAT THE QUADRUPOLE TRACE $Q_{k k}$ is constant in time}

The time derivative of the quadrupole trace $Q_{k k}$ is given by Eq. (20) as

$$
\dot{Q}_{k k}=-\iint d^{2} \mathbf{r}\left(\frac{r^{2}}{2}-\frac{\lambda^{2}}{2}\right) \frac{\partial}{\partial x_{i}}\left(u_{i} \omega\right)=\iint d^{2} \mathbf{r} x_{i} u_{i} \omega
$$

We again introduce the stream function $\psi$, and write

$$
\psi=\hat{\psi}+\widetilde{\psi},
$$

where $\hat{\psi}$ is the stream function associated with the monopole term in the series (7) and $\widetilde{\psi}$ is the stream function associated with all the remaining terms. We have

$$
\hat{\psi}=C_{1} \ln r
$$

and

$$
\widetilde{\psi} \rightarrow C_{2} \frac{f(\theta)}{r} \quad \text { as } r \rightarrow \infty,
$$

which is important for the integrations by parts that follow. Equation (B1) becomes

$$
\begin{aligned}
\dot{Q}_{k k}= & -\varepsilon_{i j} \iint d^{2} \mathbf{r} x_{i} \frac{\partial \hat{\psi}}{\partial x_{j}} \frac{\partial^{2} \hat{\psi}}{\partial x_{m} \partial x_{m}} \\
& -\varepsilon_{i j} \iint d^{2} \mathbf{r} x_{i} \frac{\partial \hat{\psi}}{\partial x_{j}} \frac{\partial^{4} \tilde{\psi}}{\partial x_{m} \partial x_{m}} \\
& -\varepsilon_{i j} \iint d^{2} \mathbf{r} x_{i} \frac{\partial \tilde{\psi}}{\partial x_{j}} \frac{\partial^{2} \hat{\psi}}{\partial x_{m} \partial x_{m}} \\
& -\varepsilon_{i j} \iint d^{2} \mathbf{r} x_{i} \frac{\partial \tilde{\psi}}{\partial x_{j}} \frac{\partial^{2} \tilde{\psi}}{\partial x_{m} \partial x_{m}} .
\end{aligned}
$$

Since $\hat{\psi}$ is a function of $r$ only, the first two integrands on the right-hand side of Eq. (B5) depend on the symmetric tensor $x_{i} x_{j}$; the integrals are thus symmetric in $i$ and $j$, and the contraction with the antisymmetric tensor $\varepsilon_{i j}$ implies that the first two integrals on the right-hand side of Eq. (B5) vanish.

The third integral on the right-hand side of Eq. (B5) is

$$
\begin{aligned}
& -\varepsilon_{i j} \iint d^{2} \mathbf{r} x_{i} \frac{\partial \tilde{\psi}}{\partial x_{j}} \frac{\partial^{2} \hat{\psi}}{\partial x_{m} \partial x_{m}} \\
& =\varepsilon_{i j} \iint d^{2} \mathbf{r} \frac{\partial \tilde{\psi}}{\partial x_{j}} x_{i} \delta^{\lambda}=-\varepsilon_{i j} \iint d^{2} \mathbf{r} \tilde{\psi} \frac{\partial}{\partial x_{j}}\left(x_{i} \delta^{\lambda}\right) \\
& =-\varepsilon_{i j} \iint d^{2} \mathbf{r} \tilde{\psi}\left(\delta_{i j} \delta^{\lambda}+x_{i} x_{j} \frac{\delta^{\lambda^{\prime}}}{r}\right),
\end{aligned}
$$

which is again zero due to the contraction of an antisymmetric tensor with two symmetric tensors.

The fourth integral on the right-hand side of Eq. (B5) is

$$
\begin{aligned}
-\varepsilon_{i j} \iint d^{2} \mathbf{r} x_{i} \frac{\partial \tilde{\psi}}{\partial x_{j}} \frac{\partial^{2} \tilde{\psi}}{\partial x_{m} \partial x_{m}} \\
=\varepsilon_{i j} \iint d^{2} \mathbf{r} \tilde{\psi} \frac{\partial}{\partial x_{j}}\left(x_{i} \frac{\partial^{2} \tilde{\psi}}{\partial x_{m} \partial x_{m}}\right) \\
=\varepsilon_{i j} \iint d^{2} \mathbf{r} \tilde{\psi}\left(\delta_{i j} \frac{\partial^{2} \tilde{\psi}}{\partial x_{m} \partial x_{m}}+x_{i} \frac{\partial^{3} \tilde{\psi}}{\partial x_{j} \partial x_{m} \partial x_{m}}\right) \\
=\varepsilon_{i j} \iint d^{2} \mathbf{r} \tilde{\psi} x_{i} \frac{\partial^{3} \tilde{\psi}}{\partial x_{j} \partial x_{m} \partial x_{m}} \\
=\varepsilon_{i j} \iint d^{2} \mathbf{r} \frac{\partial^{2}}{\partial x_{m} \partial x_{m}}\left(\tilde{\psi} x_{i}\right) \frac{\partial \tilde{\psi}}{\partial x_{j}}=\varepsilon_{i j} \iint d^{2} \mathbf{r}\left(2 \frac{\partial \tilde{\psi}}{\partial x_{m}} \delta_{i m}\right. \\
\left.+\frac{\partial^{2} \tilde{\psi}}{\partial x_{m} \partial x_{m}} x_{i}\right) \frac{\partial \tilde{\psi}}{\partial x_{j}}=2 \varepsilon_{m j} \iint d^{2} \mathbf{r} \frac{\partial \tilde{\psi}}{\partial x_{m}} \frac{\partial \tilde{\psi}}{\partial x_{j}}
\end{aligned}
$$

Nagem et al.: Vorticity dynamics and sound generation 


$$
+\varepsilon_{i j} \iint d^{2} \mathbf{r} x_{i} \frac{\partial^{2} \tilde{\psi}}{\partial x_{m} \partial x_{m}} \frac{\partial \tilde{\psi}}{\partial x_{j}}=\varepsilon_{i j} \iint d^{2} \mathbf{r} x_{i} \frac{\partial^{2} \tilde{\psi}}{\partial x_{m} \partial x_{m}} \frac{\partial \tilde{\psi}}{\partial x_{j}}
$$

The final line of Eq. (B7) states that the fourth integral on the right-hand side of Eq. (B5) is equal to the negative of itself, and is therefore zero. We have now shown that each of the four terms on the right-hand side of Eq. (B5) vanishes, and that $Q_{k k}$ is constant in time.

${ }^{1}$ M. J. Lighthill, "On sound generated aerodynamically," Proc. R. Soc. London, Ser. A 211, 564-586 (1952).
${ }^{2}$ M. S. Howe, Theory of Vortex Sound (Cambridge University Press, New York, 2003).

${ }^{3}$ H. Lamb, Hydrodynamics (Dover, New York, 1945), pp. 591-592.

${ }^{4}$ T. Gallay and C. E. Wayne, "Invariant manifolds and the long-time asymptotics of the Navier-Stokes and vorticity equations on $\mathbf{R}^{2}$," Arch. Ration. Mech. Anal. 163, 209-258 (2002).

${ }^{5} \mathrm{H}$. Grad, "Note on $N$-dimensional hermite polynomials," Commun. Pure Appl. Math. 2, 325-330 (1949).

${ }^{6}$ A. Sommerfeld, Mechanics of Deformable Bodies (Academic, New York, 1964), pp. 154-161.

${ }^{7}$ A. Powell, "Theory of vortex sound," J. Acoust. Soc. Am. 36, 177-195 (1964).

${ }^{8}$ B. E. Mitchell, S. K. Lele, and P. Moin, "Direct computation of the sound from a compressible co-rotating vortex pair," J. Fluid Mech. 285, 181-202 (1995). 
Copyright of Journal of the Acoustical Society of America is the property of American Institute of Physics and its content may not be copied or emailed to multiple sites or posted to a listserv without the copyright holder's express written permission. However, users may print, download, or email articles for individual use. 